\title{
CCR9 as a prognostic marker and therapeutic target in hepatocellular carcinoma
}

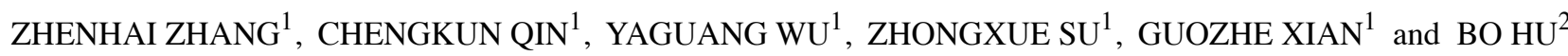 \\ Departments of ${ }^{1}$ Hepatobiliary Surgery and ${ }^{2}$ Emergency, Shandong Provincial Hospital \\ Affiliated to Shandong University, Jinan, Shandong 250021, P.R. China
}

Received November 30, 2013; Accepted January 7, 2014

DOI: $10.3892 /$ or.2014.2998

\begin{abstract}
The chemokine receptor CCR9 was recently implicated in tumor biology. In the present study, our objective was to evaluate the clinical significance and potential role of CCR9 in hepatocellular carcinoma (HCC). CCR9 expression was detected by immunohistochemistry, quantitative PCR (qPCR) and western blotting in HCC patients. The prognostic significance of CCR9 expression was assessed. The functional roles of CCR9 in HCC were investigated using MTT, BrdU, colony formation assay and flow cytometry. CCR9 was significantly elevated in HCC tissue samples. High CCR9 expression was correlated with multiple tumor nodes, high Edmondson-Steiner grade and vascular invasion. Multivariate analysis showed that CCR9 expression was an independent prognostic factor for the overall survival (OS) of HCC patients. Further investigations revealed that ectopic expression of CCR9 enhanced cell proliferation and tumorigenicity in HCC cells, whereas CCR9 silencing impaired cell proliferation and tumorigenicity, which was mediated through downregulation of the cell cycle regulators p21, p27 as well as upregulation of cyclin D1. These results suggest that CCR9 can act as a novel prognostic marker and therapeutic target for HCC.
\end{abstract}

\section{Introduction}

Hepatocellular carcinoma (HCC) is the most common type of liver cancer, comprising $90 \%$ of primary liver cancers (1). In the past decade, HCC has become one of the most frequent tumors and the most lethal cancer worldwide (2). More than $80 \%$ of HCC cases are from the Asian and African continents, and $>50 \%$ of cases are from mainland China with a vast majority of viral hepatitis patients (3). Recently, increasing trends in HCC incidence were reported from several Western countries, including France, Australia and the USA (3). Despite new advances in the management of HCC, treatment

Correspondence to: Dr Bo Hu, Emergency Department, Shandong Provincial Hospital Affiliated to Shandong University, 324 Jing Wu Road, Jinan, Shandong 250021, P.R. China

E-mail: drhubo@163.com

Key words: CCR9, hepatocellular carcinoma, prognosis, marker, therapeutic target of advanced-stage HCC remains a challenge owing to the complex nature of the disease and the lack of available therapies (4). Long-term survival after hepatic resection remains very poor for the majority of HCC patients who develop recurrence or metastasis (5). Therefore, targeting invasion and metastasis is an attractive strategy for HCC therapy. Exploring the signaling pathways implicated in the pathogenesis of HCC is a crucial step in the development of new therapeutic strategies for HCC (6).

Chemokines are a class of small inflammatory or homeostatic cytokines sharing a common biological activity in stimulating the migration of different types of cells including lymphocytes, monocytes, neutrophils, endothelial cells, mesenchymal stem cells and malignant epithelial cells (7). Chemokines/chemokine receptor network are critical in shaping the tumor immune response (8). The chemokine receptor CCR9 was initially identified for its role in the immune system, responsible for recruiting immune cells (9). Activation of CCR9 by its selective ligand CCL25 is associated with pancreatic cancer cell proliferation (10). CCR9 is functionally and significantly expressed in breast cancer tissue and activation of this receptor promotes breast tumor cell migration, invasion and MMP expression, which are key components of breast cancer metastasis (11). However, the role of CCR9 in HCC remains unclear. In the present study, our aim was to evaluate the expression of CCR9 in HCC and to investigate its clinical significance and potential role in the invasion and metastasis of HCC.

\section{Materials and methods}

Patient samples. A total of 240 cases of paraffin-embedded HCC samples and their matched non-HCC liver tissues which had been clinically and histologically diagnosed at the Shandong Provincial Hospital Affiliated to Shandong University (Shandong, China) from 2000 to 2004 were used. The CCR9 expression from 240 HCC samples was subjected to immunohistochemistry (IHC). All participants underwent hepatectomy with a median age of 48 years (range 29-78 years). In addition, fresh specimens with vascular invasion $(n=39)$ and without vascular invasion $(n=26)$ were also analyzed by quantitative PCR (qPCR) and western blotting. Prior written consent from patients and approval by the Institutional Ethics Committee were obtained. 
Follow-up. Follow-up data were obtained by reviewing the medical records and from direct communication with patients. Follow-up visits were scheduled postoperatively at intervals of one month for six months, bimonthly for six months, quarterly for six months, and semiannually for life. Complete follow-up, ranging from 0 to 83 months, was available for all patients and the median survival was 34 months.

IHC. Immunohistochemical analysis was carried out to study CCR9 protein expression in 240 human HCC tissues. In brief, paraffin-embedded specimens were cut into $4-\mu \mathrm{m}$ sections and baked at $65^{\circ} \mathrm{C}$ for $30 \mathrm{~min}$. The sections were deparaffinized with xylenes and rehydrated. Sections were submerged into EDTA antigenic retrieval buffer and microwaved for antigenic retrieval. The sections were treated with $3 \%$ hydrogen peroxide in methanol to quench the endogenous peroxidase activity, followed by incubation with $1 \%$ bovine serum albumin to block nonspecific binding. Rabbit anti-CCR9 (1:50; LifeSpan Biosciences) was incubated with the sections overnight at $4^{\circ} \mathrm{C}$. For negative controls, the rabbit anti-CCR9 antibody was replaced with normal goat serum, or the rabbit anti-CCR9 antibody was blocked with a recombinant CCR9 polypeptide by co-incubation at $4^{\circ} \mathrm{C}$ overnight preceding the immunohistochemical staining procedure. After washing, the tissue sections were treated with biotinylated anti-rabbit secondary antibody, followed by further incubation with streptavidin-horseradish peroxidase complex (both from LifeSpan Biosciences). The tissue sections were immersed in 3-amino-9-ethylcarbazole and counterstained with $10 \%$ Mayer's hematoxylin, dehydrated and mounted in crystal mount.

The degree of immunostaining of formalin-fixed, paraffinembedded sections was reviewed and scored independently by two observers, based on both the proportion of positively stained tumor cells and the intensity of staining $(12,13)$. The proportion of tumor cells was scored as follows: 0 (no positive tumor cells), 1 ( $<10 \%$ positive tumor cells), 2 (10-50\% positive tumor cells) and 3 (>50\% positive tumor cells). The intensity of staining was graded according to the following criteria: 0 (no staining), 1 (weak staining = light yellow), 2 (moderate staining $=$ yellow brown) and 3 (strong staining $=$ brown). The staining index was calculated as staining intensity score $\mathrm{x}$ proportion of positive tumor cells. Using this method of assessment, we evaluated the expression of CCR 9 by determining the staining index, which scores as 0-4, 6 and 9 . Cut-off values for CCR9 were chosen on the basis of a measure of heterogeneity with the log-rank test statistical analysis with respect to overall survival (OS). An optimal cut-off value was identified: the staining index score of $\geq 4$ was used to define tumors as high CCR9 expression and $\leq 3$ as low expression of CCR9.

$q P C R$. Total RNA from cells and primary tumor materials was extracted using the TRIzol reagent (Invitrogen) according to the manufacturer's instructions. The extracted RNA $(5 \mu \mathrm{g})$ from each sample was used for cDNA synthesis primed with random hexamers. Real-time PCR primers were designed using the Primer Express v 2.0 software (Applied Biosystems). The primers were: CCR9 forward, 5'-GTGCCTCCCT GAGATCATGT-3' and reverse, 5'-TGTGCTTTTGGCATCT TTTG-3'; $\beta$-actin forward, 5'-GCTGTATTCCCCTCCATC
GT-3' and reverse, 5'-GCCATGTTCAATGGGGTACT-5'. For PCR amplification of CCR9 cDNA, an initial amplification using CCR9-specific primers was performed with a denaturation step at $95^{\circ} \mathrm{C}$ for $10 \mathrm{~min}$, followed by 35 cycles of denaturation at $95^{\circ} \mathrm{C}$ for $60 \mathrm{sec}$, primer annealing at $58^{\circ} \mathrm{C}$ for $30 \mathrm{sec}$ and primer extension at $72^{\circ} \mathrm{C}$ for $30 \mathrm{sec}$. Upon completion of the cycling steps, a final extension at $72^{\circ} \mathrm{C}$ for 5 min was performed before the reaction was stored at $4^{\circ} \mathrm{C}$. Expression data were normalized to the geometric mean of housekeeping gene $\beta$-actin to control the variability in expression levels. The results were analyzed using the $\Delta \Delta \mathrm{Ct}$ method.

Western blot analysis. Total protein was extracted and determined by the Bradford assay using a commercial kit purchased from the Bio-Rad Laboratories. Equal quantities of protein were separated electrophoretically on $10 \%$ SDS/polyacrylamide gels and transferred onto polyvinylidene difluoride membranes (Roche). The membrane was probed with a 1:500-diluted anti-CCR9 antibody (Abcam). Expression of CCR9 was determined with horseradish peroxidase-conjugated anti-rabbit IgG $(1: 2,000)$ and enhanced chemiluminescence (Pierce) according to the manufacturer's suggested protocols. The membranes were stripped and reprobed with an anti- $\beta$-actin antibody (1:2,000; Sigma) as a loading control.

Cell lines and vector construction. The cell lines HepG2, Huh7, HEP3B and HCCLM3 were purchased from the American Type Culture Collection (ATCC) and were grown in DMEM (Invitrogen) supplemented with $10 \%$ fetal bovine serum (FBS) (HyClone). Overexpression of CCR9 human full-length CCR9 cDNA was amplified by PCR and cloned into a pMSCV-puro retroviral vector by OriGene Technologies (Rockville, MD, USA). For CCR9 knockdown, shRNA sequences were cloned into the pSUPER-retro-puro plasmid to generate pSUPER-retro CCR9 shRNA obtained from OriGene Technologies.

Cell proliferation and colony formation assay. The MTT assay was used to assess the cell proliferation. Briefly, cells were seeded in 96-well plates at a density of $2 \times 10^{3}$ cells/well. One plate was taken out at the same time every day after the cells adhered. Twenty microliters of MTT $(5 \mathrm{mg} / \mathrm{ml})$ were added to each well, and the cells were incubated for another $4 \mathrm{~h}$. The medium was removed and the formazan precipitate was solubilized in $150 \mathrm{ml}$ dimethyl sulfoxide. The absorbance at $490 \mathrm{~nm}$ was measured using a microplate reader. All experiments were performed in triplicate.

Regarding the colony formation, cells were seeded in 6 -well plates $\left(2 \times 10^{3}\right.$ cells/plate $)$ and cultured for 10 days. Subsequently, cells were fixed with ice-cold methanol for $10 \mathrm{~min}$, followed by staining with $1 \%$ crystal violet for $1 \mathrm{~min}$.

Cell cycle analysis using the BrdU. HCC cells were seeded on coverslips at a density of $5 \times 10^{4}$ cells and $24 \mathrm{~h}$ post-seeding, the cells were incubated with BrdU for $1 \mathrm{~h}$, followed by staining with anti-BrdU antibody (Sigma) according to the manufacturer's instructions (Roche Diagnostics). Images were captured using a laser scanning microscope (Olympus).

Anchorage-independent growth assay. The anchorage-independent growth ability was determined in soft agar, as 

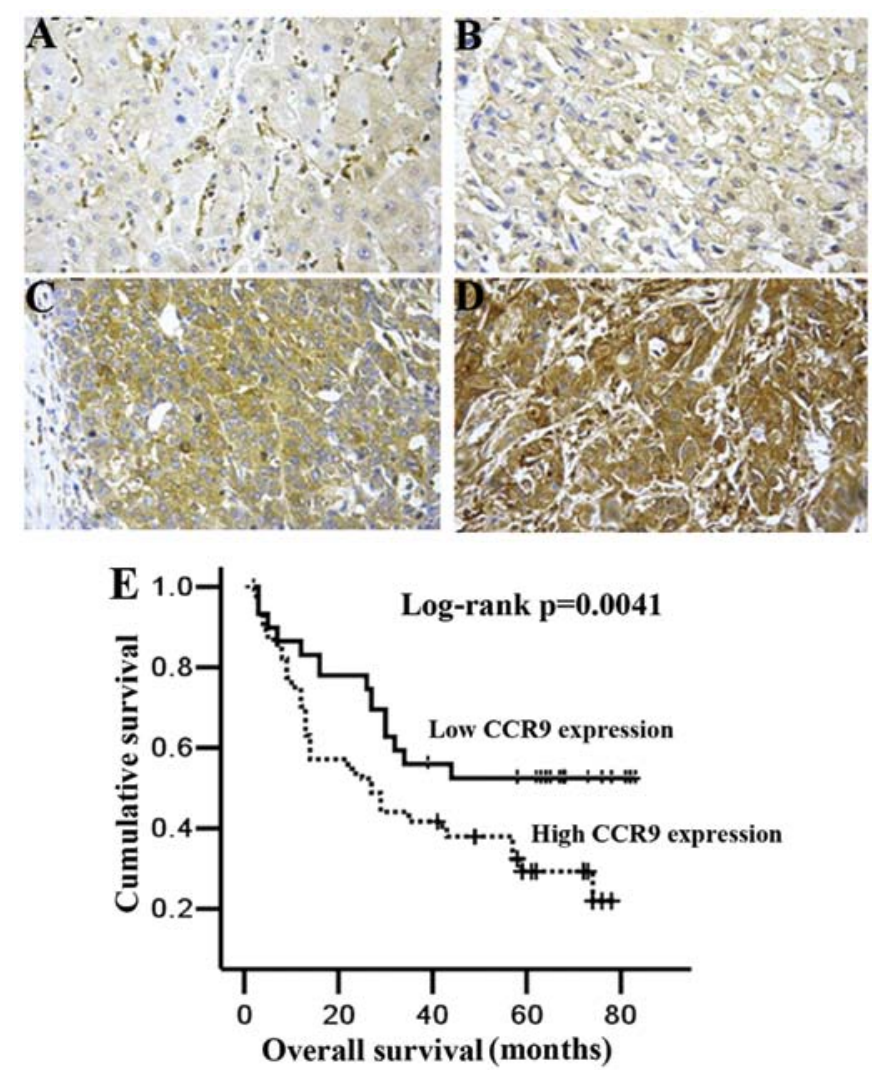

Figure 1. Association of CCR9 expression in hepatocellular carcinoma (HCC) tissues with overall survival by immunohistochemistry (magnification, x200). Immunoreactivity in CCR9 staining was localized mainly in the cytoplasm. (A) Weak expression of CCR9 in adjacent non-HCC tissue. (B) Weak expression of CCR9 in HCC. (C) Moderate expression of CCR9 in HCC. (D) Strong expression of CCR9 in HCC. (E) Overall survival was analyzed using the Kaplan-Meier method and log-rank test.

previously described (14). Briefly, $3 \mathrm{ml}$ of $0.5 \%$ agar in basal modified Eagle's medium supplemented with $10 \%$ FBS was layered onto each well of 6-well tissue culture plates. Cells ( $3 \times 10^{4}$ cells) suspended in $1 \mathrm{ml}$ normal medium were mixed with $2 \mathrm{ml}$ of $0.5 \%$ agar-basal modified Eagle's medium supplemented with $10 \%$ FBS, and $1 \mathrm{ml}$ of mixture was added into each well over the top of the $0.5 \%$ agar layer. Plates were incubated at $37^{\circ} \mathrm{C}$ in $5 \% \mathrm{CO}_{2}$ for 2 weeks, and the colonies with $>32$ cells of each were counted.

Statistical analysis. All statistical analyses were carried out using the SPSS 17.0 statistical software package. The Mann-Whitney U test was used to analyze the correlation between CCR9 expression and the clinicopathological characteristics. Survival curves were plotted using the Kaplan-Meier method and compared with the log-rank test. The significance of various variables for survival was analyzed by the Cox proportional hazards model in the univariate and multivariate analyses. $\mathrm{P}<0.05$ was considered to indicate a statistically significant difference.

\section{Results}

CCR9 expression in HCC tissues. CCR9 expression was detected mainly in the cytoplasm of tumor cells (Fig. 1).
Table I. Correlation between CCR9 expression and clinicopathological features in hepatocellular carcinoma.

\begin{tabular}{|c|c|c|c|c|}
\hline \multirow[b]{2}{*}{ Variables } & \multirow{2}{*}{$\begin{array}{c}\text { No. of } \\
\text { cases }\end{array}$} & \multicolumn{2}{|c|}{$\begin{array}{c}\text { CCR9 } \\
\text { expression }\end{array}$} & \multirow[b]{2}{*}{ P-value } \\
\hline & & Low & High & \\
\hline Gender & & & & 0.20 \\
\hline Male & 189 & 84 & 105 & \\
\hline Female & 51 & 22 & 29 & \\
\hline Age (years) & & & & 0.26 \\
\hline$\leq 60$ & 198 & 94 & 104 & \\
\hline$>60$ & 42 & 12 & 30 & \\
\hline Liver cirrhosis & & & & 0.485 \\
\hline Presence & 173 & 67 & 106 & \\
\hline Absence & 67 & 39 & 28 & \\
\hline Capsular formation & & & & 0.71 \\
\hline Presence & 125 & 61 & 66 & \\
\hline Absence & 115 & 45 & 68 & \\
\hline Tumor size & & & & 0.36 \\
\hline$\leq 5 \mathrm{~cm}$ & 89 & 36 & 53 & \\
\hline$>5 \mathrm{~cm}$ & 151 & 70 & 81 & \\
\hline Tumor nodule no. & & & & 0.009 \\
\hline Multiple ( $\geq 2)$ & 96 & 35 & 61 & \\
\hline Solitary & 144 & 71 & 73 & \\
\hline $\begin{array}{l}\text { Edmondson-Steiner } \\
\text { grade }\end{array}$ & & & & 0.012 \\
\hline Stage I-II & 131 & 62 & 69 & \\
\hline Stage III-IV & 109 & 44 & 65 & \\
\hline Vascular invasion & & & & 0.0015 \\
\hline Presence & 133 & 45 & 88 & \\
\hline Absence & 107 & 61 & 46 & \\
\hline
\end{tabular}

Clinicopathological variables were compared between the group with low CCR9 expression $(n=106)$ and the group with high CCR9 expression $(n=134)$.

According to the classification described in the Materials and methods, immunohistochemical analysis clearly showed that high CCR9 expression was present in 55.8\% (134 of 240) of HCC tissues. In the non-HCC tissues, we observed high CCR9 protein expression only in $9.6 \%$ (23 of 240) $(\mathrm{P}<0.01)$. These results indicated that CCR9 expression was significantly elevated in HCC samples.

CCR9 expression correlates with clinicopathological features and prognosis in HCC patients. Immunohistochemical analysis showed that the high CCR9 levels correlated with tumor node number, high Edmondson-Steiner grade and vascular invasion (Table I). As indicated in the Materials and methods, 240 HCC tissues were divided into the group with high CCR9 expression $(n=134)$ and the group with low CCR9 expression $(n=106)$. As a result, HCC patients with high CCR9 expression had markedly reduced $\mathrm{OS}(\mathrm{P}=0.0041$; Fig. 1E) compared 
Table II. Univariate and multivariate analysis showing overall survival for hepatocellular carcinoma patients.

\begin{tabular}{|c|c|c|c|c|c|c|}
\hline \multirow{2}{*}{ Variables } & \multicolumn{3}{|c|}{ Univariate analysis } & \multicolumn{3}{|c|}{ Multivariate analysis } \\
\hline & HR & $95 \% \mathrm{CI}$ & P-value & HR & $95 \% \mathrm{CI}$ & P-value \\
\hline CCR9 & 2.91 & $1.76-6.50$ & 0.001 & 2.35 & $1.09-5.02$ & 0.014 \\
\hline Gender & 0.62 & $0.28-1.52$ & 0.59 & & & \\
\hline Age & 0.82 & $0.48-1.37$ & 0.74 & & & \\
\hline Tumor size & 1.69 & $1.13-3.43$ & 0.017 & 1.43 & $0.98-2.07$ & 0.072 \\
\hline Histologic grade & 1.36 & $0.86-1.96$ & 0.20 & & & \\
\hline Cirrhosis & 0.69 & $0.41-1.14$ & 0.14 & & & \\
\hline HBsAg status & 1.44 & $0.56-2.73$ & 0.58 & & & \\
\hline Serum AFP & 1.32 & $0.92-2.74$ & 0.09 & & & \\
\hline Metastasis & 1.30 & $0.73-2.70$ & 0.30 & & & \\
\hline Recurrence & 1.22 & $0.80-2.26$ & 0.25 & & & \\
\hline
\end{tabular}

HR, hazard ratio; CI, confidence interval; AFP, $\alpha$-fetoprotein.
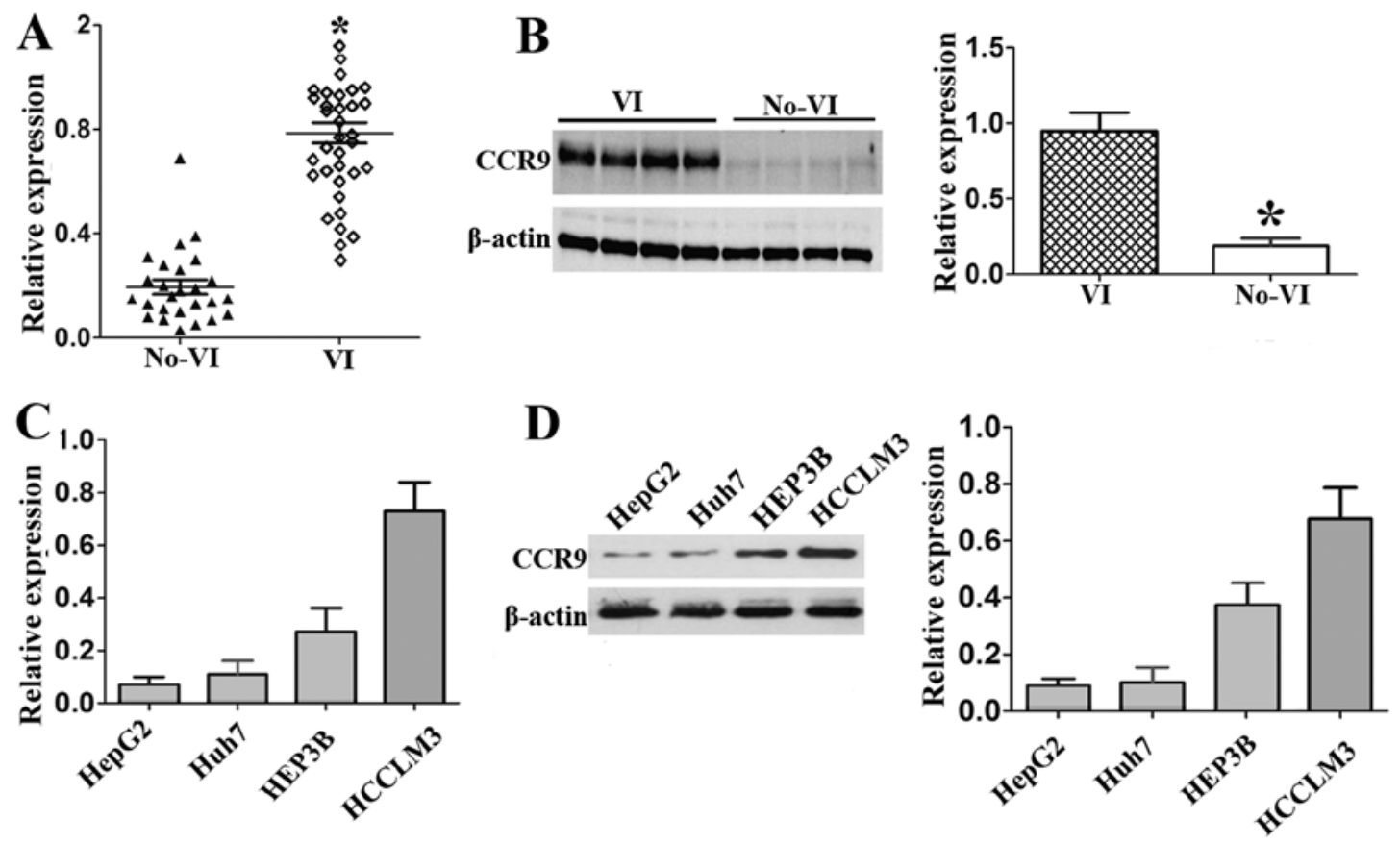

Figure 2. CCR9 expression level in hepatocellular carcinoma (HCC) tissues and HCC cell lines. (A) CCR9 mRNA expression level in HCC tissues with vascular invasion (VI) and without VI (no-VI) by real-time RT-PCR. $\beta$-actin was used as internal control. (B) CCR9 protein expression level in HCC tissues with VI and without VI (no-VI) by western blotting. $\beta$-actin was used as loading control. (C) The mRNA and (D) protein expression of CCR9 in four different HCC cell lines (HepG2, Huh7, HEP3B and HCCLM3) was also detected."P $<0.01$.

with patients with low CCR9 expression. Multivariate analysis showed that CCR9 was an independent prognostic factor for the OS of HCC patients (Table II, hazard ratio=2.35, 95\% confidence interval=1.09-5.02, $\mathrm{P}=0.014)$. These findings support that CCR9 plays an important role in HCC.

Correlation between CCR9 expression and vascular invasion. We also investigated the relationship between CCR9 expression and vascular invasion in fresh HCC tissues using qPCR and western blotting. The results showed that CCR9 mRNA level in HCC tissues with vascular invasion was significantly higher than in tissues without vascular invasion $(\mathrm{P}<0.01$, Fig. 2A). Consistent with the mRNA expression, the CCR9 protein expression level was also significantly elevated in HCC tissues with vascular invasion $(\mathrm{P}<0.01$; Fig. $2 \mathrm{~B})$.

Furthermore, we detected the expression levels of CCR9 in HCC cell lines. Among the four HCC cell lines, HCCLM3, with the highly metastatic ability (15), had the highest CCR9 expression at mRNA and protein levels, followed by HEP3B, Huh7 and HepG2 cells (Fig. 2C and D). These data suggest that there may be a correlation between CCR9 and the metastasis potential of HCC. 
A
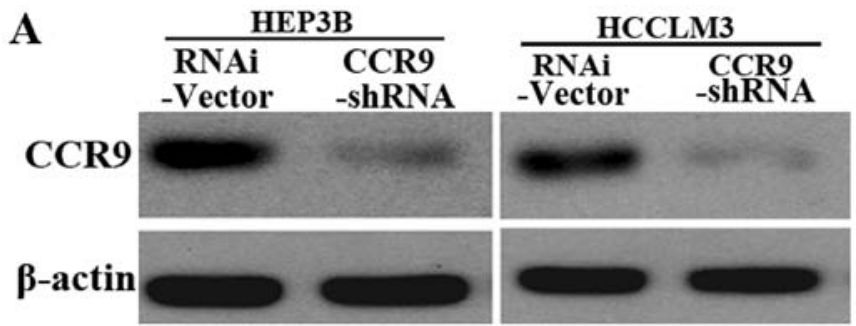

B
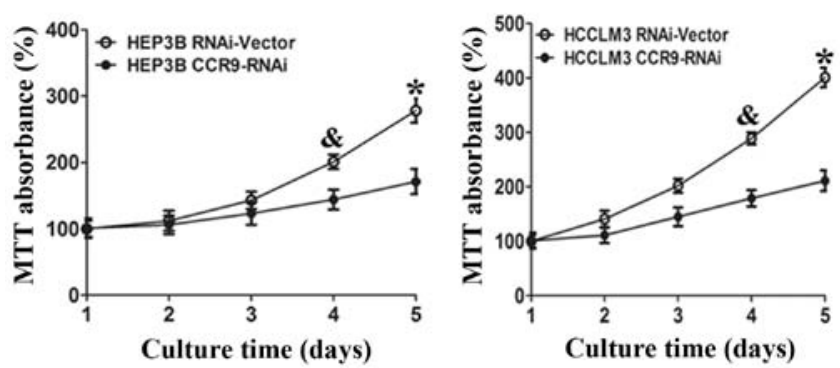

\section{C}
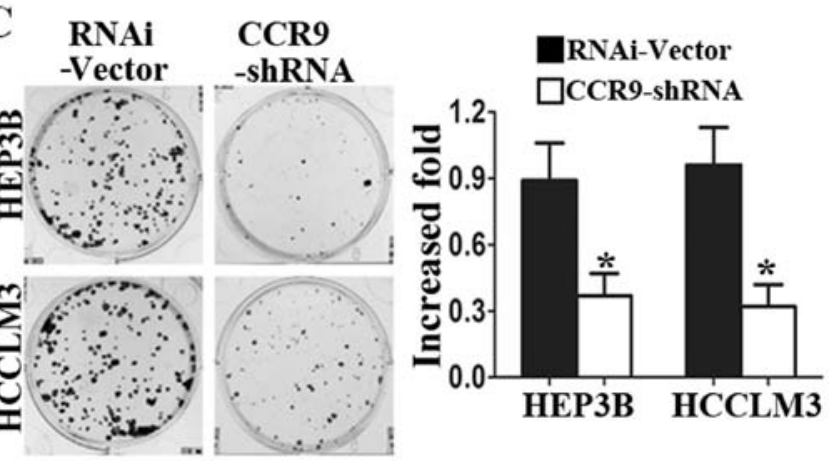

Figure 3. CCR9 knockdown suppresses hepatocellular carcinoma (HCC) cell proliferation. (A) Western blot analysis of CCR9 expression in HCC cell lines stably infected with CCR9-shRNA. $\beta$-actin was used as a loading control. (B) MTT assay analysis of cell growth rates at the indicated times after seeding cells. (C) Representative images (left panel) and quantification (right panel) of colony formation in CCR9-silenced and vector cells. Data are means \pm SD from three independent experiments. ${ }^{\&} \mathrm{P}<0.05,{ }^{*} \mathrm{P}<0.01$.

Knockdown of CCR9 inhibits the proliferation of HEP3B and HCCLM3 cells. To further investigate the potential role of CCR9 in HCC, we assessed the effects of CCR9 silencing on the cell proliferation of HCC cell lines. Based on the CCR9 expression levels, we chose HEP3B and HCCLM3 as the study cells.

Western blot analysis showed that CCR9 expression in the two cell lines were knocked down (Fig. 3A). MTT assays showed significant reduction in cell proliferation of HEP3BshCCR9 and HCCLM3-shCCR9 cells compared with control cells at 4 and 5 days (Fig. 3B). Silencing of CCR9 also reduced the colony formation abilities (Fig. 3C) in both cell lines. Herein, we showed that CCR9 silencing inhibited the proliferation of HCCLM3 and HEP3B cells compared with control cells.

Overexpression of CCR9 promotes the proliferation of $\mathrm{HCC}$ cells. Furthermore, we investigated the potential role of CCR9 overexpression in HCC cell lines. Western blot analysis showed that stable CCR9-overexpressed cell lines were established (Fig. 4A). MTT assays showed a significant increase
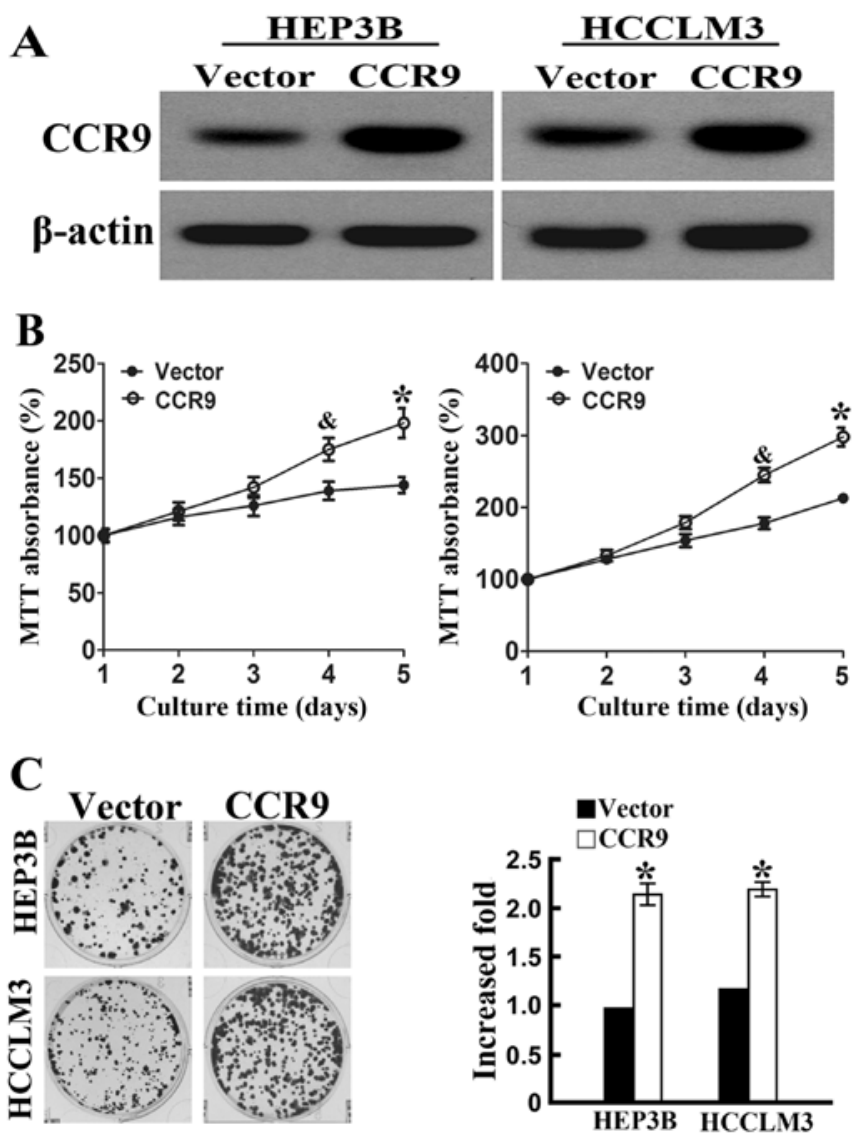

Figure 4. CCR9 overexpression promotes hepatocellular carcinoma (HCC) cell proliferation. (A) Western blot analysis of CCR9 expression in HCC cell lines with stably overexpressed CCR9. $\beta$-actin was used as a loading control. (B) MTT assay analysis of cell growth rates at the indicated times after seeding cells. (C) Representative images (left panel) and quantification (right panel) of colony formation in CCR9-overexpressed and vector cells. Data are means \pm SD from three independent experiments. ${ }^{\circledR} \mathrm{P}<0.05,{ }^{*} \mathrm{P}<0.01$.

in cell proliferation of $\mathrm{HEP} 3 \mathrm{~B}^{\mathrm{CCR} 9+}$ cells and HCCLM3 ${ }^{\mathrm{CCR} 9+}$ cells compared with control cells at 4 and 5 days (Fig. 4B). Overexpression of CCR 9 also increased the colony formation abilities (Fig. 4C) in the two cell lines. Therefore, we showed that CCR9 overexpression promoted the proliferation of HEP3B and HCCLM3 cells compared with control cells.

CCR9 promotes cell proliferation by increasing the ratio of HCC cells at $S$ phase. Since CCR9 promoted cell proliferation, we further explored the potential mechanisms. The percentage of cells at $\mathrm{S}$ phase was assessed by immunodetection of BrdU. Ectopic expression of CCR9 markedly increased the percentage of BrdU-positive cells in HCC cell lines; 29.4 vs. $43.5 \%$ for HEP3B and 27.2 vs. $41.8 \%$ for HCCLM3 (Fig. 5A). Conversely, CCR9 silencing significantly decreased the $S$ phase fraction of BrdU-positive cells; 32.27 vs. $17.03 \%$ for HEP3B cells and 31.23 vs. $17.98 \%$ for HCCLM3 cells (Fig. 5B). Flow cytometric analysis of cell cycle also confirmed that CCR9 overexpression markedly increased the percentage of cells at $\mathrm{S}$ phase and decreased the percentage of cells in the G1/G0 phase (Fig. 5C). In contrast, CCR 9 knockdown increased the percentage of cells in the G1/G0 phase and decreased the percentage of cells at $\mathrm{S}$ phase (Fig. 5D). These results showed that CCR9 

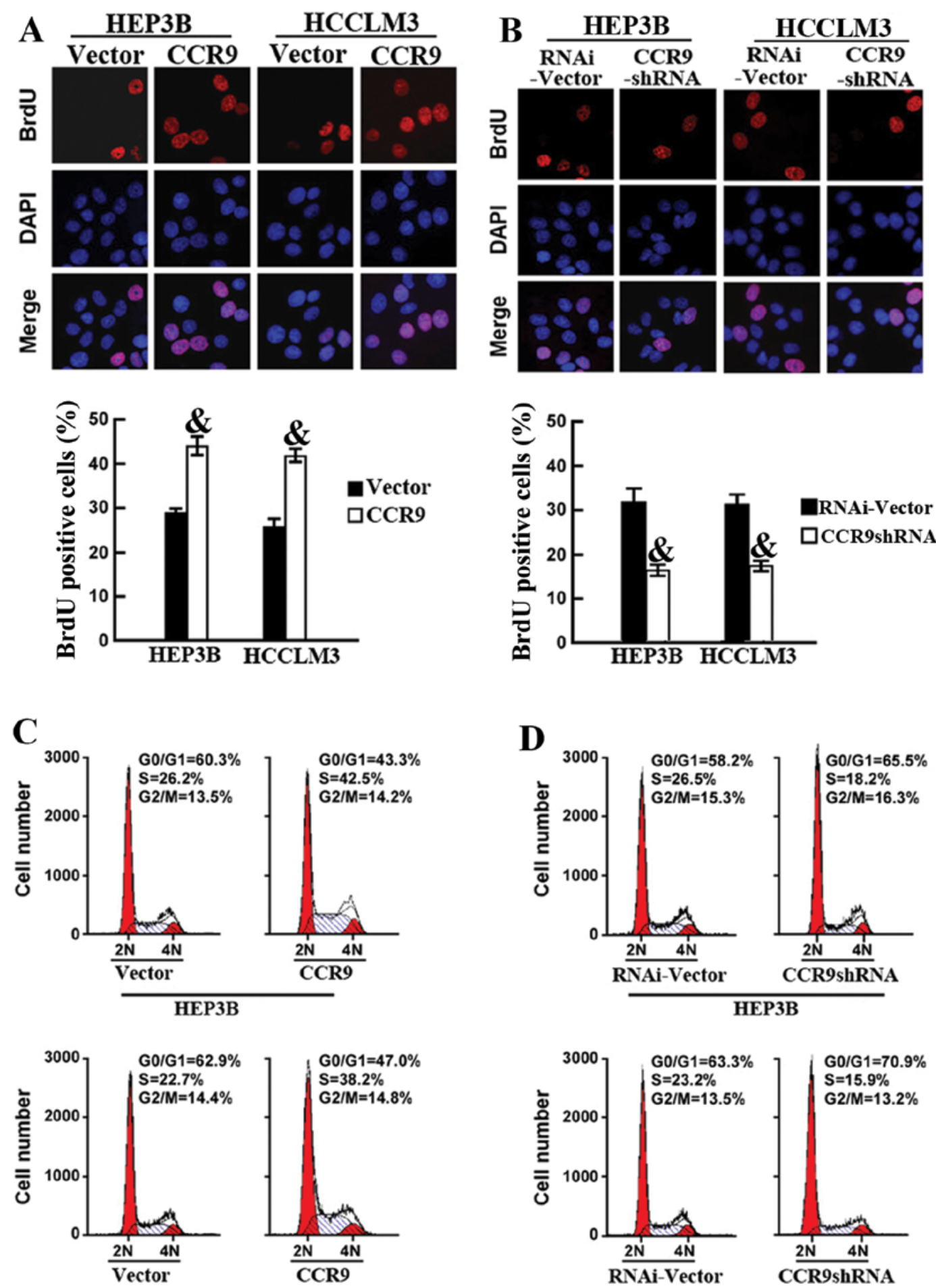

HCCLM3

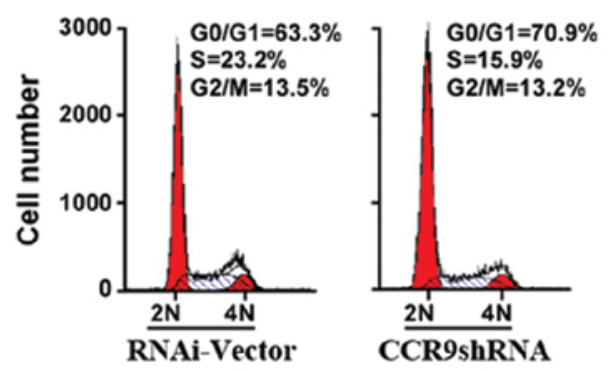

HCCLM3

Figure 5. CCR9 promotes the cell proliferation by increasing the fraction of HCC cells at S phase. (A) Representative images (upper) and quantification (lower) of BrdU incorporation in CCR9-overexpressed and vector cells. (B) Representative images (upper) and quantification (lower) of BrdU incorporation in CCR9silenced and vector cells. (C) Flow cytometric analysis of CCR9-overexpressed and vector cells. (D) Flow cytometric analysis of CCR9-silenced and vector cells. Data are means $\pm \mathrm{SD}$ from three independent experiments. ${ }^{\circledR} \mathrm{P}<0.05$.

promoted cell proliferation by increasing the fraction of $\mathrm{HCC}$ cells at $\mathrm{S}$ phase.

CCR 9 promotes cell proliferation via regulation of $p 21, p 27$ and cyclin D1. The p21, p27 and cyclin D1 play key roles in the control of cell cycle associated with tumorigenicity $(16,17)$.
qPCR showed that CCR9 overexpression significantly reduced p21 and p27, and increased cyclin D1 (Fig. 6A). In contrast, CCR9 silencing markedly enhanced p21 and p27 expression, and inhibited cyclin D1 expression in both cell lines (Fig. 6B). These results indicated that CCR9 regulates p21, p27 and cyclin D1 to promote cell proliferation and tumorigenicity. 

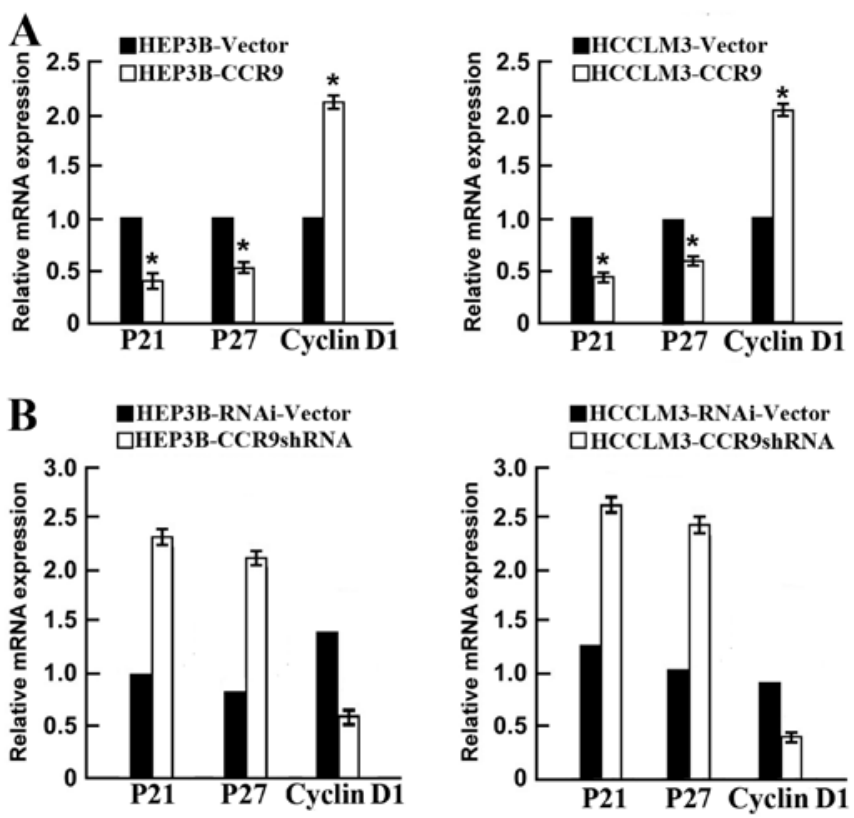

Figure 6.CCR9 modulates p21,p27 and cyclin D1 expression in hepatocellular carcinoma (HCC) cells by real-time qRT-PCR. (A) Overexpression of CCR9 downregulated p21 and p27 and upregulated cyclin D1. (B) Knockdown of CCR9 upregulated p21 and p27 and downregulated cyclin D1. Data were normalized to $\beta$-actin control and are expressed as means \pm SD from three independent experiments. ${ }^{*} \mathrm{P}<0.05$.

\section{Discussion}

Recent data show that $\mathrm{CCR} 9^{+}$macrophages are required for acute liver inflammation in mouse models of hepatitis (18) and may activate hepatic stellate cells, promoting liver fibrosis in mice (19). Our results from the present study show that CCR9 was significantly elevated in HCC tissues. The increased CCR9 expression was correlated with aggressive features of HCC. Moreover, survival analysis revealed that high CCR9 expression was associated with poor OS in HCC. Multivariate analysis revealed that CCR9 was an independent factor for predicting survival in $\mathrm{HCC}$.

The chemokine receptor CCR9 was initially identified for its role in the immune system, where it is present on leukocytes and is critical in T cell development (9). Subsequent data indicated that CCR9 expression is associated with increased cancer cell invasiveness in melanoma, ovarian, breast and prostate cancer (20-22). CCR9 shows aberrant expression on pancreatic cancer cells (10) and may be a factor in promoting pancreatic cancer progression. While the CCL25/CCR9 axis has been examined in some types of cancer, it remains unknown whether CCR9 plays a role in HCC. In the present study, we investigated interactions between HCC cell invasiveness and CCR9 expression. We found that CCR9 overexpression increased the fraction of HCC cells at $\mathrm{S}$ phase, thereby promoting cell growth. Further investigation indicated that this effect of CCR9 was mediated by regulating p21 and p27 expression as well as cyclin D1 expression at the mRNA level.

In the present study, we found that upregulation of CCR9 markedly increased proliferation of both HCC cell lines, whereas silencing of CCR9 reduced it. Furthermore, soft-agar assay revealed that the anchorage-independent HCC cell growth was significantly enhanced upon CCR9 overexpression and inhibited in case of CCR9 knockdown, suggesting that CCR9 overexpression promotes the tumorigenicity of HCC cells. Therefore, the biological roles of CCR9 in HCC metastasis merit further investigation.

In summary, the present study showed that CCR9 expression was markedly elevated in HCC tissue samples and cell lines, compared to normal control. CCR9 was demonstrated to be a novel prognostic marker for HCC. Further investigations revealed that ectopic expression of CCR9 enhanced cell proliferation in HCC cells, whereas CCR9 silencing impaired cell proliferation, which was mediated through downregulation of the cell cycle regulators p21, p27 as well as upregulation of cyclin D1. The results suggest that CCR9 may be a novel target in HCC treatment. Future studies are warranted to expand our understanding of CCR9-mediated signaling in HCC and to develop novel therapeutic agents to target this pathway in HCC.

\section{Acknowledgements}

This study was supported by the Natural Science Foundation of Shandong Province (no. ZR2012HM079), China.

\section{References}

1. Zhu AX, Duda DG, Sahani DV and Jain RK: HCC and angiogenesis: possible targets and future directions. Nat Rev Clin Oncol 8: 292-301, 2011.

2. Cervello M, McCubrey JA, Cusimano A, Lampiasi N, Azzolina A and Montalto G: Targeted therapy for hepatocellular carcinoma: novel agents on the horizon. Oncotarget 3: 236-260, 2012.

3. McClune AC and Tong MJ: Chronic hepatitis B and hepatocellular carcinoma. Clin Liver Dis 14: 461-476, 2010.

4. Finn RS: Emerging targeted strategies in advanced hepatocellular carcinoma. Semin Liver Dis 33 (Suppl 1): S11-S19, 2013.

5. Zhou L, Liu J and Luo F: Serum tumor markers for detection of hepatocellular carcinoma. World J Gastroenterol 12: 1175-1181, 2006.

6. Psyrri A, Arkadopoulos N, Vassilakopoulou M, Smyrniotis V and Dimitriadis G: Pathways and targets in hepatocellular carcinoma. Expert Rev Anticancer Ther 12: 1347-1357, 2012.

7. Viola A and Luster AD: Chemokines and their receptors: drug targets in immunity and inflammation. Annu Rev Pharmacol Toxicol 48: 171-197, 2008.

8. Franciszkiewicz K, Boissonnas A, Boutet $\mathrm{M}$, Combadière $\mathrm{C}$ and Mami-Chouaib F: Role of chemokines and chemokine receptors in shaping the effector phase of the antitumor immune response. Cancer Res 72: 6325-6332, 2012.

9. Svensson M and Agace WW: Role of CCL25/CCR9 in immune homeostasis and disease. Expert Rev Clin Immunol 2: 759-773, 2006.

10. Shen X, Mailey B, Ellenhorn JD, Chu PG, Lowy AM and Kim J: CC chemokine receptor 9 enhances proliferation in pancreatic intraepithelial neoplasia and pancreatic cancer cells. J Gastrointest Surg 13: 1955-1962, 2009.

11. Johnson-Holiday C, Singh R, Johnson E, et al: CCL25 mediates migration, invasion and matrix metalloproteinase expression by breast cancer cells in a CCR9-dependent fashion. Int J Oncol 38: 1279-1285, 2011.

12. Geisler SA, Olshan AF, Weissler MC, et al: p16 and p53 protein expression as prognostic indicators of survival and disease recurrence from head and neck cancer. Clin Cancer Res 8: 3445-3453, 2000.

13. Fukuoka J, Fuji T, Shih JH, et al: Chromatin remodeling factors and BRM/BRG1 expression as prognostic indicators in non-small cell lung cancer. Clin Cancer Res 10: 4314-4324, 2004.

14. Cao Z, Zhang R, Li J, et al: X-linked inhibitor of apoptosis protein (XIAP) regulation of cyclin D1 protein expression and cancer cell anchorage-independent growth via its E3 ligasemediated protein phosphatase $2 \mathrm{~A} / \mathrm{c}-\mathrm{Jun}$ axis. J Biol Chem 288: 20238-20247, 2013. 
15. Ye QH, Qin LX, Forgues M, et al: Predicting hepatitis B virus-positive metastatic hepatocellular carcinomas using gene expression profiling and supervised machine learning. Nat Med 9: 416-423, 2003.

16. Yu Y, Kovacevic $Z$ and Richardson DR: Tuning cell cycle regulation with an iron key. Cell Cycle 6: 1982-1994, 2007.

17. Macaluso M, Montanari M, Cinti C and Giordano A: Modulation of cell cycle components by epigenetic and genetic events. Semin Oncol 32: 452-457, 2005.

18. Nakamoto N, Ebinuma H, Kanai T, et al: $\mathrm{CCR}^{+}$macrophages are required for acute liver inflammation in mouse models of hepatitis. Gastroenterology 142: 366-376, 2012.
19. Chu PS, Nakamoto N, Ebinuma H, et al: C-C motif chemokine receptor 9 positive macrophages activate hepatic stellate cells and promote liver fibrosis in mice. Hepatology 58: 337-350, 2013.

20. Amersi FF, Terando AM, Goto Y, et al: Activation of CCR9/ CCL25 in cutaneous melanoma mediates preferential metastasis to the small intestine. Clin Cancer Res 14: 638-645, 2008.

21. Johnson EL, Singh R, Singh S, et al: CCL25-CCR9 interaction modulates ovarian cancer cell migration, metalloproteinase expression, and invasion. World J Surg Oncol 8: 62, 2010.

22. Singh S, Singh UP, Stiles JK, Grizzle WE and Lillard JW Jr: Expression and functional role of CCR9 in prostate cancer cell migration and invasion. Clin Cancer Res 10: 8743-8750, 2004. 